\title{
Two-Level Linear Programming Problems with Two Decision-Makers at the Upper Level: An Interactive Fuzzy Approach
}

\author{
M. Borza ${ }^{1}$, A. S. Rambely ${ }^{1} \&$ M. Saraj ${ }^{2}$ \\ ${ }^{1}$ School of Mathematical Sciences, Faculty of Science \& Technology, Universiti Kebangsaan Malaysia, 43600 \\ UKM Bangi, Selangor, Malaysia \\ ${ }^{2}$ Department of Mathematics, Faculty of Mathematical Sciences \& Computer, Shahid Chamran University, \\ Ahvaz, Iran \\ Correspondence: M. Borza, School of Mathematical Sciences, Faculty of Science \& Technology, Universiti \\ Kebangsaan Malaysia, 43600 UKM Bangi, Selangor, Malaysia. E-mail: mborza@siswa.ukm.edu.my
}

Received: April 21, $2014 \quad$ Accepted: June 3, $2014 \quad$ Online Published: June 30, 2014

doi:10.5539/mas.v8n4p211 URL: http://dx.doi.org/10.5539/mas.v8n4p211

\begin{abstract}
The interactive fuzzy programming approach can be used to address two-level programming problems if a mutually cooperative relationship exists between the decision-makers. In this approach, a satisfactory solution is obtained by taking into account the minimum satisfaction level of the decision-maker at the upper level. In addition, the overall satisfaction balance between the decision-maker at the lower level and the decision-maker at the upper level must be appropriate. In this paper, interactive fuzzy programming is used to achieve a satisfactory solution for a two-level linear programming problem with two decision-makers at the upper level. The method is designed in such a way that both decision-makers at the upper level achieve their minimum satisfaction levels together with the appropriate satisfaction balance between the decision-maker at the lower level and each decision-maker at the upper level. A numerical example is given to illustrate the method. Moreover, it is indicated that a three-level program can be considered as a two-level program with two decision-makers at the upper level.
\end{abstract}

Keywords: membership function, satisfactory solution, optimization, interactive fuzzy programming, multi-level programming

\section{Introduction}

Two-level mathematical programming problems are used to model decision-making problems in the real scenarios of decentralized organizations (Abd El-Wahed \& Lee, 2006; Bard, 1998; Migdalas et al., 1998; Sakawa et al., 2001, 2002; Stackelberg, 1934). In the two-level programming problem, two decision-makers (DMs) make decisions successively. The DM at the upper level (leader) specifies a strategy; then the DM at the lower level (follower) subsequently specifies a strategy to optimize his or her own objective function with full knowledge of the leader's action. Finally, the leader optimizes his or her own objective function according to the rational response of the follower. The obtained solution described in this situation is a Stackelberg solution. Three categories are considered for obtaining Stackelberg solutions to the two-level linear programming problems (two-level LPPs) as the vertex enumeration approach (Bialas \& Karwan, 1984), the Kuhn-Tucker approach (Bard \& Falk, 1982; Bard \& Moore, 1990; Bialas \& Karwan, 1984; Hansen et al., 1992) and the penalty function approach (Anandalingam \& White, 1990; White \& Anandalingam, 1993).

The concept of the Stackelberg solution arises when no cooperative relationship exists between the DMs, or if these DMs do not come to an agreement even if such a cooperative relationship exists. The problem of finding a Stackelberg solution is well known and this problem is both non-convex and strongly NP hard.

Lai (1996) and Shih et al. (1996) proposed the use of fuzzy programming to obtain a solution if a cooperative relationship exists between DMs, a situation that differs from the Stackelberg solution concept. In their fuzzy approach, the membership function of the objective functions and the variables are used to obtain a satisfactory solution. Because of the inconsistency between the membership functions of variables and objective functions, this method does not always yield a desirable solution. Later, Sakawa et al. (1998), suggested an interactive fuzzy programming approach in which only the membership of the objective functions plays role in obtaining a 
satisfactory solution. In this approach, the leader first specifies a minimum level of satisfaction, which is updated during the algorithm to achieve a reasonable overall satisfaction balance between the leader and the follower. Eventually, the method leads to a compromise satisfactory solution, which is also a Pareto optimal solution.

The application of the interactive fuzzy programming approach is not limited to two-level programming problems, but can be used to address multi-level programming problems, decentralized two-level programming problems, nonlinear two-level programming problems, and fuzzy two-level linear fractional programming problems (Sakawa et al., 1998, Sakawa \& Nishizaki, 2001, Sakawa \& Nishizaki, 2002, Sakawa et al., 2000, Borza et al. 2012). All of these problems have been solved with the assumption that only one DM exists at the upper level.

For a decentralized organization with three DMs the appropriate mathematical model for the organization represents a three-level programming problem with single DM at each level, or a two-level programming problem with two DMs at the upper level and single DM at the lower level; or a two-level programming problem with single DM at the upper level and two DMs at the lower level.

Bard (1984) and Wen and Bialas (1986) introduced approaches to address non-cooperative three-level linear programming problems. In the method of Bard (1984), the Stackelberg solution obtained using Kuhn-Tucker optimal conditions. While, in the method of Wen and Bialas (1986), the Stackelberg solution obtained using Kth best method. The non-cooperative two-level LPPs with single leader and many followers were considered by Simaan and Cruz (1973), and Anandalingam (1988). In the proposed methods, the leader optimizes the objective of self over a feasible region which is constructed by the intersection of the inducible regions made by the followers individually. The analysis of the Stackelberg solutions to the non-cooperative two-level LPPs with many leaders and single follower was studied by Sherali (1984).

The interactive fuzzy programming approaches proposed by Lai (1996), Shih et al. (1996), Sakawa et al. (1998), and Sakawa and Nishizaki (2009) can be used to find satisfactory solutions to the cooperative three-level linear programming problems. In addition, Sakawa and Nishizaki (2002) introduced an interactive fuzzy programming approach to address the cooperative two-level LPP with single leader and multiple followers. In their interactive fuzzy programming, the satisfactory solutions are obtained after passing two phases.

Under these circumstances, this paper aims to introduce an interactive fuzzy programming approach so as to obtain a satisfactory solution for the two-level LPPs with two DMs at the upper level and single DM at the lower level when a mutually cooperative relationship exists between the DMs. In the method, the minimum satisfaction levels of the leaders are updated during the algorithm to achieve an overall satisfaction balance between the follower and each leader. A numerical example is also given to illustrate the method. Additionally, it is showed that a three-level LPP can be transformed into a two-level LPP with two DMs at the upper level and single DM at the lower level. As a consequence, the proposed method can be used instead of rather difficult method introduced by Sakawa and Nishizaki (2009).

\section{Method}

The general form of a two-level LPP with two DMs at the upper level and a single DM at the lower level with a cooperative relationship established among the DMs is formulated as follows:

Problem 1

$$
\begin{aligned}
& \underset{\text { upper level }}{\text { Minimize }} z_{1}\left(x_{1}, x_{2}, x_{3}\right)=c_{11} x_{1}+c_{12} x_{2}+c_{13} x_{3}, \\
& \underset{\text { upper level }}{\text { Minimize }} z_{2}\left(x_{1}, x_{2}, x_{3}\right)=c_{21} x_{1}+c_{22} x_{2}+c_{23} x_{3}, \\
& \text { Minimize } \text { lower level }_{3} z_{3}\left(x_{1}, x_{2}, x_{3}\right)=c_{31} x_{1}+c_{32} x_{2}+c_{33} x_{3}, \\
& \text { s.t } \quad A_{1} x_{1}+A_{2} x_{2}+A_{3} x_{3} \leq b, \\
& \quad x_{1} \geq 0, x_{2} \geq 0, x_{3} \geq 0,
\end{aligned}
$$

where $x_{i}, i=1,2,3$ is an $n_{i}$-dimensional decision variable, $c_{i 1}, i=1,2,3$ is an $n_{1}$-dimensional constant row vector, $c_{i 2}, i=1,2,3$ is an $n_{2}$-dimensional constant row vector, $c_{i 3}, i=1,2,3$ is an $n_{3}$-dimensional constant row vector, $b$ is an $m$-dimensional constant column vector, and $A_{i}, i=1,2,3$ is an $m \times n_{i}$ constant matrix.

In the above problem, $z_{1}\left(x_{1}, x_{2}, x_{3}\right), z_{2}\left(x_{1}, x_{2}, x_{3}\right)$, and $z_{3}\left(x_{1}, x_{2}, x_{3}\right)$, respectively, represent objective functions of the upper levels, and the lower level, while $x_{1}, x_{2}$, and $x_{3}$, respectively, represent decision variables of the 
upper levels, and the lower level.

First, to apply the interactive fuzzy programming approach to obtain a satisfactory solution to Problem 1, every DM must define a membership function according to his or her own fuzzy goals. In this paper, we assume that DMi for $i=1,2,3$ selects the following linear membership function $\mu_{i}\left(z_{i}\left(x=\left(x_{1}, x_{2}, x_{3}\right)\right)\right)$, which is a strictly monotonic decreasing function for $z_{i}^{\min } \leq z_{i}(x) \leq z_{i}{ }^{\max }$.

$$
\mu_{i}\left(z_{i}(x)\right)=\left\{\begin{array}{cc}
0, & z_{i}(x) \geq z_{i}^{\max } \\
\frac{z_{i}(x)-z_{i}^{\max }}{z_{i}{ }^{\text {min }}-z_{i}{ }^{\max }}, & z_{i}^{\min } \leq z_{i}(x) \leq z_{i}^{\max } \\
1, & z_{i}(x) \leq z_{i}^{\min }
\end{array}\right.
$$

In the above definition, $z_{i}^{\text {min }}$ and $z_{i}^{\text {max }}$ are fuzzy goals for $i^{\text {th }}$ decision-maker, and can be specified by the following roles:

$$
\text { Minimize }\left\{z_{i}(x): x \in X\right\} \quad \text { for } i=1,2,3 \text {. }
$$

Let $x^{i}$ for $i=1,2,3$ be the solution of the $i^{\text {th }}$ problems in (2); subsequently, to find $z_{i}{ }^{\text {max }}$ for $i=1,2,3$ the suggested method by Zimmermann (1978) can be used as follows:

$$
z_{i}^{\max }=\operatorname{Maximize}\left\{z_{i}\left(x^{j}\right) \text { for } j=1,2,3 j \neq i\right\} \text {, for } i=1,2,3 \text {. }
$$

According to the fuzzy decision making theory by Bellman and Zadeh (1970), the following addresses Problem 1:

$$
\begin{array}{ll}
\text { Maximize } & \left\{\text { minimum } \mu_{i}\left(z_{i}(x)\right) \text { for } i=1,2,3\right\} \\
\text { s.t } & A_{1} x_{1}+A_{2} x_{2}+A_{3} x_{3} \leq b, \\
& x_{1} \geq 0, x_{2} \geq 0, x_{3} \geq 0 .
\end{array}
$$

The above problem can be transformed into the following equivalent problem using the auxiliary variable $\lambda$ as follows:

Problem 2

$$
\begin{gathered}
\text { Maximize } \quad \lambda \\
\text { s.t } \left.\quad z_{1}(x)\right) \geq \lambda, \\
\mu_{2}\left(z_{2}(x)\right) \geq \lambda, \\
\mu_{3}\left(z_{3}(x)\right) \geq \lambda, \\
0 \leq \lambda \leq 1, \\
A_{1} x_{1}+A_{2} x_{2}+A_{3} x_{3} \leq b, \\
x_{1} \geq 0, x_{2} \geq 0, x_{3} \geq 0 .
\end{gathered}
$$

If both decision-makers at the upper level are satisfied with the optimal solution $x^{*}$ of the above problem, then it is concluded that $x^{*}$ becomes a satisfactory solution; otherwise, DM1 and DM2 specify the minimum of the decision-makers' satisfaction levels with full knowledge of the membership function value for decision-maker at the lower level. If $\hat{\delta}_{1}$ and $\hat{\delta}_{2}$ are the minimum satisfaction levels specified by DM1 and DM2, respectively, then the following problem must be solved to obtain a solution for which DM1 and DM2 are satisfied.

Problem 3

$$
\begin{gathered}
\text { Maximize } \mu_{3}\left(z_{3}(x)\right) \\
\mu_{1}\left(z_{1}(x)\right) \geq \hat{\delta}_{1}, \\
\mu_{2}\left(z_{2}(x)\right) \geq \hat{\delta}_{2}, \\
A_{1} x_{1}+A_{2} x_{2}+A_{3} x_{3} \leq b, \\
x_{1} \geq 0, x_{2} \geq 0, x_{3} \geq 0 .
\end{gathered}
$$

With the assumption that the objective functions at both levels conflict with one another, the obtained satisfaction level for DM1 and DM2 at $x^{*}$ causes the satisfaction level of the DM3 to decrease, and consequently, this reduction may not be desirable for the DM3 at the lower level, who acts in cooperation with DM1 and DM2. To

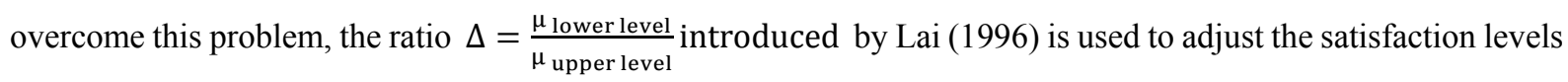


between the DMs for two-level linear programming problems with single decision-maker at both levels.

In Problem 1, because two decision-makers are presented at the upper level, according to the idea of Lai (1996), the two ratios $\Delta_{31}=\frac{\mu_{3}\left(z_{3}(x)\right)}{\mu_{1}\left(z_{1}(x)\right)}$ and $\Delta_{32}=\frac{\mu_{3}\left(z_{3}(x)\right)}{\mu_{2}\left(z_{2}(x)\right)}$ must be defined for solution $x$ to adjust the satisfaction levels between the DMs for a two-level linear programming problem with two decision-makers at the upper level and single DM at the lower level. Let $\left[\Delta_{\mathrm{L} 31}, \Delta_{\mathrm{U} 31}\right]$ and $\left[\Delta_{\mathrm{L} 32}, \Delta_{\mathrm{U} 32}\right]$ be the desirable domains for $\Delta_{31}$ and $\Delta_{32}$ specified by DM1 and DM2, respectively and $x^{*}$ be the optimal solution obtained of Problem 3. If $\Delta_{31} \in$ $\left[\Delta_{\mathrm{L} 31}, \Delta_{\mathrm{U} 31}\right]$ and $\Delta_{32} \in\left[\Delta_{\mathrm{L} 32}, \Delta_{\mathrm{U} 32}\right], x^{*}$ becomes a satisfactory solution and the interactive fuzzy process stops. Otherwise, the values of $\hat{\delta}_{1}$ and $\hat{\delta}_{2}$ must be updated. The following procedure, which is derived directly from Lai (1996), needs to be considered to update the values of $\hat{\delta}_{1}$ and $\hat{\delta}_{2}$,

[Procedure 1 to update the values of $\hat{\delta}_{1}$ and $\hat{\delta}_{2}$ ]

If no feasible solution exists for Problem 3 for minimal satisfaction levels $\hat{\delta}_{1}$ and $\hat{\delta}_{2}$, then DM1 and DM2 decrease their value of $\hat{\delta}_{1}$ and $\hat{\delta}_{2}$, respectively.

If $\Delta_{31}<\Delta_{\mathrm{L} 31}$ and $\Delta_{32} \in\left[\Delta_{\mathrm{L} 32}, \Delta_{\mathrm{U} 32}\right]$, the value of $\hat{\delta}_{1}$ is increased by DM1.

If $\Delta_{31}>\Delta_{\mathrm{U} 31}$ and $\Delta_{32} \in\left[\Delta_{\mathrm{L} 32}, \Delta_{\mathrm{U} 32}\right]$, DM1 decreases the value of $\hat{\delta}_{1}$.

If $\Delta_{32}<\Delta_{\mathrm{L} 32}$ and $\Delta_{31} \in\left[\Delta_{\mathrm{L} 31}, \Delta_{\mathrm{U} 31}\right]$, DM2 increases the value of $\hat{\delta}_{2}$.

If $\Delta_{32}>\Delta_{\mathrm{U} 32}$ and $\Delta_{31} \in\left[\Delta_{\mathrm{L} 31}, \Delta_{\mathrm{U} 31}\right]$, DM2 decreases the value of $\hat{\delta}_{2}$.

If $\Delta_{31}<\Delta_{\mathrm{L} 31}$ and $\Delta_{32}<\Delta_{\mathrm{L} 32}$, DM1 and DM2 increase the values of $\hat{\delta}_{1}$ and $\hat{\delta}_{2}$, respectively.

If $\Delta_{31}>\Delta_{\mathrm{U} 31}$ and $\Delta_{32}>\Delta_{\mathrm{U} 32}$, the values of $\hat{\delta}_{1}$ and $\hat{\delta}_{2}$ are decreased by DM1 and DM2, respectively.

If $\Delta_{31}<\Delta_{\mathrm{L} 31}$ and $\Delta_{32}>\Delta_{\mathrm{U} 32}$, the value of $\hat{\delta}_{1}$ is increased by DM1and the value of $\hat{\delta}_{2}$ is decreased by DM2.

If $\Delta_{31}>\Delta_{\mathrm{U} 31}$ and $\Delta_{32}<\Delta_{\mathrm{L} 32}$, the value of $\hat{\delta}_{1}$ is decreased by DM1 and the value of $\hat{\delta}_{2}$ is increased by DM2.

Because of many comparisons, using Procedure 1 may be encompasses some difficulties in practice.

Introducing an easier procedure is the main idea of this paper. To do this, with the assumption that $x^{*}$ is the optimal solution of Problem 3, we subsequently define:

$$
\begin{gathered}
{\left[\Delta_{L}, \Delta_{U}\right]=\left[\Delta_{L 31}, \Delta_{U 31}\right] \cap\left[\Delta_{L 32}, \Delta_{U 32}\right],} \\
\Delta_{\max }=\frac{\mu_{3}\left(z_{3}\left(x^{*}\right)\right)}{\min \left\{\mu_{1}\left(z_{1}\left(x^{*}\right)\right), \mu_{2}\left(z_{2}\left(x^{*}\right)\right)\right\}}, \\
\Delta_{\min }=\frac{\mu_{3}\left(z_{3}\left(x^{*}\right)\right)}{\max \left\{\mu_{1}\left(z_{1}\left(x^{*}\right)\right), \mu_{2}\left(z_{2}\left(x^{*}\right)\right)\right\}} .
\end{gathered}
$$

Remark 1. It is inferred from definitions of $\Delta_{\max }^{l}$ and $\Delta_{\min }^{l}$ that $\Delta_{31} \in\left[\Delta_{\mathrm{L} 31}, \Delta_{\mathrm{U} 31}\right]$ and $\Delta_{32} \in\left[\Delta_{\mathrm{L} 32}, \Delta_{\mathrm{U} 32}\right]$ if and only if $\Delta_{\text {max }}^{l} \in\left[\Delta_{L}, \Delta_{U}\right]$ and $\Delta_{\text {min }}^{l} \in\left[\Delta_{L}, \Delta_{U}\right]$.

In accordance with the values of $\Delta_{\max }$ and $\Delta_{\min }$ obtained from relations (5) and (6), DM1 and DM2 must update levels of their satisfactions, according to the following procedure:

[Procedure 2 to update the minimal satisfactory levels $\hat{\delta}_{1}$ and $\hat{\delta}_{2}$ ]

If no feasible solution exists for Problem 3 for a minimal satisfaction level $\hat{\delta}_{1}$ and $\hat{\delta}_{2}$, then DM1 and DM2 decrease their value of $\hat{\delta}_{1}$ and $\hat{\delta}_{2}$, respectively.

If $\Delta_{\max }>\Delta_{U}$, then the decision-maker at the upper level with a minimum value of membership function must increase his or her own minimum satisfaction level.

If $\Delta_{\min }<\Delta_{L}$, then the decision-maker at the upper level with a maximum value of the membership function must decrease his or her minimum satisfaction level.

If $\Delta_{\max }<\Delta_{L}$, then both decision-makers at the upper level must decrease the values of their minimum satisfaction level.

If $\Delta_{\min }>\Delta_{u}$, then both decision-makers at the upper level must increase the values of their minimum satisfaction 
level.

For the updated values $\hat{\delta}_{1}$ and $\hat{\delta}_{2}$, Problem 3 must be resolved and the ratios $\Delta_{\max }$ and $\Delta_{\min }$ must be checked again for the obtained optimal solution. Additionally, the above procedure needs to be repeated until the values of $\Delta_{\text {max }}$ and $\Delta_{\text {min }}$ lie in the interval $\left[\Delta_{L}, \Delta_{U}\right]$.

At iteration $l$, let $\mu_{1}\left(z_{1}^{l}\right), \mu_{2}\left(z_{2}^{l}\right), \mu_{3}\left(z_{3}^{l}\right), \Delta_{\max }^{l}=\frac{\mu_{3}\left(z_{3}^{l}\right)}{\min \left\{\mu_{1}\left(z_{1}^{l}\right), \mu_{2}\left(z_{2}^{l}\right)\right\}}$ and $\Delta_{\min }^{l}=\frac{\mu_{3}\left(z_{3}^{l}\right)}{\max \left\{\mu_{1}\left(z_{1}^{l}\right), \mu_{2}\left(z_{2}^{l}\right)\right\}}$ denote the degrees of satisfaction of DM1, DM2 and DM3, the ratio of satisfaction degree of the follower to the minimum satisfaction degree of the leaders, and the ratio of satisfaction degree of the follower to the maximum value of satisfaction degree of the leaders, respectively. Let the solution be $x^{l}$ at iteration $l$. The interactive process terminates if the following two conditions are satisfied, and DM1 and DM2 yield the overall satisfactory solution. [Termination conditions of the interactive process]

Condition 1: The minimal satisfaction levels of DM1 and DM2 must be greater than $\hat{\delta}_{1}$ and $\hat{\delta}_{2}$, respectively. (i.e., $\mu_{1}\left(z_{1}^{l}\right) \geq \hat{\delta}_{1}$ and $\left.\mu_{2}\left(z_{2}^{l}\right) \geq \hat{\delta}_{2}\right)$.

Condition 2: $\Delta_{\text {max }}^{l} \in\left[\Delta_{L}, \Delta_{U}\right]$ and $\Delta_{\text {min }}^{l} \in\left[\Delta_{L}, \Delta_{U}\right]$.

Proposition 1. Solution obtained of the interactive fuzzy programming introduced in the above is a Pareto optimal solution.

Proof. Let $x^{l}$ be an unique optimal solution of Problem 3obtained in iteration $l$. If $x^{l}$ is not a Pareto optimal solution for Problem 1. Therefore, there exists feasible point $\tilde{x}$ such that $z_{j}(\tilde{x})<z_{j}\left(x^{l}\right)$ for some $j$ and $z_{i}(\tilde{x}) \leq z_{i}\left(x^{*}\right)$, for $i=1,2,3, i \neq j$. Due to the fact that $\mu_{i}\left(z_{i}(x)\right)$ is a monotone decreasing function for $z_{i}(x)$, accordingly, we have:

$\mu_{i}\left(z_{i}(\tilde{x})\right) \geq \mu_{i}\left(z_{i}\left(x^{l}\right)\right) \geq \hat{\delta}_{i}$, for $i=1,2,3, i \neq j$ and $\mu_{j}\left(z_{j}(\tilde{x})\right)>\mu_{j}\left(z_{j}\left(x^{l}\right)\right) \geq \hat{\delta}_{j}$. This is a contradiction to uniqueness optimality of $x^{l}$ for Problem 3 .

Remark 2. The following problem needs to be considered to check the uniqueness of $x^{l}$ obtained from Problem 3. (Sakawa 1993).

$$
\begin{gathered}
\text { Maximize } \epsilon_{1}+\epsilon_{2}+\epsilon_{3} \\
\text { s.t } \\
z_{1}(x)+\epsilon_{1} \leq z_{1}\left(x^{l}\right), \\
z_{2}(x)+\epsilon_{2} \leq z_{2}\left(x^{l}\right), \\
z_{3}(x)+\epsilon_{3} \leq z_{3}\left(x^{l}\right), \\
A_{1} x_{1}+A_{2} x_{2}+A_{3} x_{3} \leq b, \\
\epsilon_{1} \geq 0, \epsilon_{2} \geq 0, \epsilon_{3} \geq 0, x_{1} \geq 0, x_{2}, x_{3} \geq 0 .
\end{gathered}
$$

Let an optimal solution of the above problem be $(\bar{x}, \bar{\epsilon})$ where $\bar{\epsilon}=\left(\bar{\epsilon}_{1}, \bar{\epsilon}_{2}, \bar{\epsilon}_{3}\right)$. If $\bar{\epsilon}=0$ then $x^{l}$ is a Pareto optimal solution for Problem 3, otherwise $\bar{x}$ is a Pareto optimal solution.

\section{Numerical Example}

The following two-level linear programming problem with two decision-makers at the upper level is solved to illustrate the proposed method. All data are taken from Sakawa and Nishizaki (2009).

Problem 4

$$
\begin{aligned}
& \min _{\text {upper level }} \sum_{i=1}^{15} c_{1 i} x_{i} \\
& \min _{\text {upper level }} \sum_{i=1}^{15} c_{2 i} x_{i} \\
& \min _{\text {lower level }} \sum_{i=1}^{15} c_{3 i} x_{i} \\
& \text { s.t } \quad A_{1} x_{1}+\cdots+A_{15} x_{15} \leq b, \\
& x_{i} \geq 0 \text { for } i=1, \ldots, 15 \text {. }
\end{aligned}
$$


Table 1. The coefficients of Problem 4

\begin{tabular}{|c|c|c|c|c|c|c|c|c|c|c|c|c|c|c|c|c|c|}
\hline$i$ & 1 & 2 & 3 & 4 & 5 & 6 & 7 & 8 & 9 & 10 & 11 & 12 & 13 & 14 & 15 & & \\
\hline$c_{1 i}$ & -49 & -5 & -45 & -5 & -34 & -16 & -33 & -19 & -12 & -21 & -7 & -23 & -12 & -8 & -11 & & \\
\hline$c_{2 i}$ & -12 & -10 & -4 & -19 & -10 & -30 & -25 & -49 & -18 & -37 & -17 & -13 & -24 & -15 & -10 & & \\
\hline$c_{3 i}$ & -25 & -7 & -31 & -12 & -24 & -12 & -5 & -4 & -33 & -6 & -41 & -22 & -37 & -14 & -23 & & \\
\hline \multirow[t]{16}{*}{$\boldsymbol{A}_{\boldsymbol{i}}$} & 46 & -29 & -48 & 31 & 21 & -47 & -37 & 37 & 32 & 19 & 21 & 2 & -11 & -35 & -2 & $b$ & 0 \\
\hline & 0 & 39 & 12 & -14 & 29 & -42 & -26 & 42 & 31 & -19 & -25 & 6 & 4 & 2 & 5 & & 26 \\
\hline & 38 & -27 & 5 & -31 & 14 & -38 & -29 & -5 & -47 & 49 & -45 & -45 & 5 & 10 & -40 & & -111 \\
\hline & -26 & 16 & 44 & 6 & 19 & 17 & 27 & 32 & 17 & -6 & -27 & 1 & 18 & -6 & 15 & & 88 \\
\hline & -48 & 13 & 2 & -33 & 19 & 22 & -35 & 27 & -35 & -35 & -26 & -16 & 37 & 47 & -2 & & -37 \\
\hline & 9 & -6 & 12 & -17 & -32 & -8 & 24 & -24 & 45 & -31 & 16 & -9 & -19 & 17 & 44 & & 12 \\
\hline & -9 & 2 & -16 & 8 & 32 & -6 & -25 & -25 & -8 & 4 & 23 & 41 & 30 & 36 & 11 & & 45 \\
\hline & 24 & 30 & 42 & -26 & 16 & 19 & -18 & -18 & 9 & -34 & -46 & 30 & 3 & -1 & -45 & & -8 \\
\hline & -26 & -8 & 0 & 41 & -42 & -19 & 13 & -42 & 49 & -27 & 4 & -2 & -12 & 24 & -33 & & -47 \\
\hline & -29 & 16 & -16 & -4 & 18 & 45 & -8 & 21 & 6 & 47 & 43 & 46 & 26 & 22 & -5 & & 136 \\
\hline & -7 & 1 & -3 & 38 & 18 & -43 & -15 & 31 & -34 & 23 & -35 & -34 & 20 & -15 & -26 & & -48 \\
\hline & -12 & 4 & 47 & 0 & -4 & -18 & -19 & 28 & 47 & -36 & -45 & 20 & 40 & 3 & -15 & & 19 \\
\hline & -12 & -46 & 11 & -47 & -47 & 19 & 30 & 50 & 12 & -24 & 13 & 20 & -43 & -8 & 20 & & -31 \\
\hline & 5 & -2 & 37 & 38 & 0 & 12 & -34 & 34 & 28 & -40 & -18 & 33 & 39 & 14 & 2 & & 88 \\
\hline & 49 & 41 & 3 & 12 & -48 & 15 & 12 & 32 & 31 & -28 & -25 & -23 & -6 & -25 & -15 & & 14 \\
\hline & -17 & -6 & 34 & 21 & 11 & 5 & -28 & -46 & -15 & 9 & 12 & 49 & 4 & -17 & -47 & & -18 \\
\hline
\end{tabular}

To identify the membership functions of the fuzzy goals for the objective functions, three individual minimization problems of the three decision-makers are solved at the beginning of the procedure. The individual minima and the corresponding optimal solution are shown in Table 2.

Table 2. Optimal Solutions to individual problems

\begin{tabular}{lllllllllllllllll}
\hline & $x_{1}$ & $x_{2}$ & $x_{3}$ & $x_{4}$ & $x_{5}$ & $x_{6}$ & $x_{7}$ & $x_{8}$ & $x_{9}$ & $x_{10}$ & $x_{11}$ & $x_{12}$ & $x_{13}$ & $x_{14}$ & $x_{15}$ & $z_{i}^{\min }$ \\
\hline DM1 & 3.18 & 0 & 0 & 1 & 3.46 & 2.24 & 4.08 & 0 & 0 & 0.30 & 1.56 & 0 & 0 & 0.93 & 0 & -475 \\
DM2 & 2.72 & 0 & 0 & 2.22 & 2.56 & 1.64 & 4.41 & 0 & 0 & 1.05 & 1.28 & 0 & 0 & 1.55 & 0 & -345 \\
DM3 & 2.63 & 0 & 0.88 & 0.61 & 2.41 & 1.61 & 3.09 & 0 & 0 & 0.26 & 2.21 & 0 & 1.1 & 0 & 0 & -327 \\
\hline
\end{tabular}

Suppose that the decision-makers employ the linear membership function (1) whose parameters are determined by relations (2) and (3). Subsequently, one finds that $\left(z_{1}^{\min }, z_{1}^{\max }\right)=(-474.6844,-414.4563),\left(z_{2}^{\min }, z_{2}^{\max }\right)=$ $(-344.4466,-296.4661)$ and $\left(z_{3}^{\min }, z_{3}^{\max }\right)=(-327.4543,-279.0825)$, and maximization of Problem 2 for this problem can be written as:

Problem 5

\section{Maximize $\lambda$}

s.t

$$
\begin{gathered}
\left(z_{1}(x)+414.4563\right) /(-474.6844+414.4563) \geq \lambda \\
\left(z_{2}(x)+296.4661\right) /(-344.4466+296.4661) \geq \lambda \\
\left(z_{3}(x)+279.0825\right) /(-327.4543+279.0825) \geq \lambda \\
x \in S
\end{gathered}
$$

where $S$ denotes the feasible region of Problem 4. The result of the first iteration, including an optimal solution to Problem 5, is shown in Table 3. 
Table 3. Iteration 1

\begin{tabular}{|c|c|c|c|c|c|c|c|c|c|c|c|c|c|c|}
\hline$x_{1}$ & $x_{2}$ & $x_{3}$ & $x_{4}$ & $x_{5}$ & $x_{6}$ & $x_{7}$ & $x_{8}$ & $x_{9}$ & $x_{10}$ & $x_{11}$ & $x_{12}$ & $x_{13}$ & $x_{14}$ & $x_{15}$ \\
\hline 2.71 & 0 & 0.73 & 2.06 & 2.42 & 1.73 & 3.57 & 0 & 0 & 0.91 & 1.67 & 0 & 0 & 1.24 & 0 \\
\hline$z_{1}^{1}$ & $z_{2}^{1}$ & $z_{3}^{1}$ & $\mu_{1}\left(z_{1}^{1}\right)$ & $\mu_{2}\left(z_{2}^{1}\right)$ & $\mu_{3}\left(z_{3}^{1}\right)$ & & & & & & & & & \\
\hline-444 & -320.3 & -303.1 & 0.498 & 0.498 & 0.498 & & & & & & & & & \\
\hline
\end{tabular}

Suppose that DM1 and DM2 are not satisfied with the solution obtained in iteration 1, taking into account the result of the first iteration, they subsequently specify their minimum satisfaction levels at $\hat{\delta}_{1}=0.75$ and $\hat{\delta}_{2}=0.7$. Moreover, suppose that DM1 and DM2 specify $\left[\Delta_{L 31}, \Delta_{U 31}\right]=[0.6,0.8]$ and $\left[\Delta_{L 32}, \Delta_{U 32}\right]=$ $[0.6,0.75]$, taking into account the result of the first iteration. According to relation (4), $\left[\Delta_{L}, \Delta_{U}\right]=[0.6,0.75]$.

The problem with the minimum satisfaction levels (3) is written as follows:

Problem 6

$$
\text { Maximize } \mu_{3}\left(z_{3}(x)\right)
$$

S.t

$$
\begin{gathered}
\left(z_{1}(x)+414.4563\right) /(-474.6844+414.4563) \geq 0.75, \\
\left(z_{2}(x)+296.4661\right) /(-344.4466+296.4661) \geq 0.7, \\
x \in S .
\end{gathered}
$$

\begin{tabular}{|c|c|c|c|c|c|c|c|c|c|c|c|c|c|c|}
\hline$x_{1}$ & $x_{2}$ & $x_{3}$ & $x_{4}$ & $x_{5}$ & $x_{6}$ & $x_{7}$ & $x_{8}$ & $x_{9}$ & $x_{10}$ & $x_{11}$ & $x_{12}$ & $x_{13}$ & $x_{14}$ & $x_{15}$ \\
\hline 2.93 & 0.00 & 0.009 & 1.67 & 2.96 & 1.91 & 4.25 & 0 & 0 & 0.711 & 1.41 & 0 & 0 & 1.26 & 0 \\
\hline$z_{1}^{2}$ & $z_{2}^{2}$ & $z_{3}^{2}$ & $\mu_{1}\left(z_{1}^{2}\right)$ & $\mu_{2}\left(z_{2}^{2}\right)$ & $\mu_{3}\left(z_{3}^{2}\right)$ & $\Delta_{\max }^{2}$ & $\Delta_{\min }^{2}$ & & & & & & & \\
\hline-459 & -330 & -289 & 0.75 & 0.7 & 0.211 & 0.3 & 0.28 & & & & & & & \\
\hline
\end{tabular}

The result of the second iteration including an optimal solution to Problem 6 is shown in Table 4 .

Table 4. Iteration 2

In the second iteration, the satisfaction degree $\mu_{1}\left(z_{1}^{2}\right)=0.75$ of DM1 becomes equal to the minimum satisfaction level 0.75 , and $\mu_{2}\left(z_{2}^{2}\right)=0.7$ of DM2 becomes equal to the minimum satisfaction level 0.7 , but the ratio $\Delta_{\max }^{2}=0.3014$ is not in interval $[0.6,0.75]$. Therefore, this solution does not satisfy the second condition for termination of the interactive process. Because $\Delta_{\text {max }}^{2}<\Delta_{L}$, both the minimum satisfaction levels of DM1 and DM2 must be decreased. Suppose that DM1 and DM2 update their minimum satisfaction levels at

\begin{tabular}{|c|c|c|c|c|c|c|c|c|c|c|c|c|c|c|}
\hline$x_{1}$ & $x_{2}$ & $x_{3}$ & $x_{4}$ & $x_{5}$ & $x_{6}$ & $x_{7}$ & $x_{8}$ & $x_{9}$ & $x_{10}$ & $x_{11}$ & $x_{12}$ & $x_{13}$ & $x_{14}$ & $x_{15}$ \\
\hline 2.8453 & 0 & 0.3 & 1.8161 & 2.7519 & 1.8469 & 3.9527 & 0 & 0 & 0.7 & 1.5 & 0 & 0 & 1.2 & 0 \\
\hline$z_{1}^{3}$ & $z_{2}^{3}$ & $z_{3}^{3}$ & $\mu_{1}\left(z_{1}^{3}\right)$ & $\mu_{2}\left(z_{2}^{3}\right)$ & $\mu_{3}\left(z_{3}^{3}\right)$ & $\Delta_{\max }^{3}$ & $\Delta_{\min }^{3}$ & & & & & & & \\
\hline-453 & -325 & -295 & 0.65 & 0.6 & 0.344 & 0.5733 & 0.529 & & & & & & & \\
\hline
\end{tabular}
$\hat{\delta}_{1}=0.65$ and $\hat{\delta}_{2}=0.6$, respectively. Then, the problem with the revised minimal satisfactory levels (3) is subsequently solved, and the result of the third iteration is shown in Table 5.

Table 5. Iteration 3

In the third iteration, the ratio $\Delta_{\max }^{3}=0.5733$ is not in the interval $[0.6,0.75]$. Therefore, this solution does not satisfy the second condition of termination of the interactive process. Because $\Delta_{\text {max }}^{3}<\Delta_{L}$, both minimal satisfactory levels of DM1 and DM2 must be decreased. Suppose that DM1 and DM2 update their minimal satisfactory levels at $\hat{\delta}_{1}=0.62$ and $\hat{\delta}_{2}=0.58$, respectively. The problem with the revised minimal satisfactory levels (3) is subsequently solved, and the result of the fourth iteration is shown in Table 6. 
Table 6. Iteration 4

\begin{tabular}{|c|c|c|c|c|c|c|c|c|c|c|c|c|c|c|}
\hline$x_{1}$ & $x_{2}$ & $x_{3}$ & $x_{4}$ & $x_{5}$ & $x_{6}$ & $x_{7}$ & $x_{8}$ & $x_{9}$ & $x_{10}$ & $x_{11}$ & $x_{12}$ & $x_{13}$ & $x_{14}$ & $x_{15}$ \\
\hline 2.8184 & 0 & 0.4 & 1.8646 & 2.6875 & 1.8237 & 3.8769 & 0 & 0 & 0.8 & 1.5 & 0 & 0 & 1.2 & 0 \\
\hline$z_{1}^{3}$ & $z_{2}^{3}$ & $z_{3}^{3}$ & $\mu_{1}\left(z_{1}^{3}\right)$ & $\mu_{2}\left(z_{2}^{3}\right)$ & $\mu_{3}\left(z_{3}^{3}\right)$ & $\Delta_{\max }^{3}$ & $\Delta_{\min }^{3}$ & & & & & & & \\
\hline-451 & -324 & -297 & 0.62 & 0.58 & 0.37 & 0.645 & 0.6 & & & & & & & \\
\hline
\end{tabular}

In the fourth iteration, the satisfaction degree $\mu_{1}\left(z_{1}^{4}\right)=0.62$ of DM1 becomes equal to the minimum satisfaction level 0.62 , and $\mu_{2}\left(z_{2}^{4}\right)=0.58$ of DM2 becomes equal to the minimal satisfactory level 0.58 , and the ratios $\Delta_{\max }^{4}=0.6453$ and $\Delta_{\min }^{4}=0.6037$ are both in the interval $[0.6,0.75]$. Therefore, this solution satisfies the termination conditions of the interactive process, and becomes a satisfactory solution to Problem 4.

\section{Three-Level Programming}

In this section, the three-level linear programming problem is addressed using two different interactive fuzzy programming approaches.

The general form of a three-level linear programming problem is given as following:

Problem 7

$$
\begin{gathered}
\text { Minimize } \text { level 1(DM1) }_{1}\left(x_{1}, x_{2}, x_{3}\right)=c_{11} x_{1}+c_{12} x_{2}+c_{13} x_{3}, \\
\underset{\text { level 2(DM2) }}{\text { Minimize }} z_{2}\left(x_{1}, x_{2}, x_{3}\right)=c_{21} x_{1}+c_{22} x_{2}+c_{23} x_{3}, \\
\underset{\text { level 3(DM3) }}{\text { Minimize }} z_{3}\left(x_{1}, x_{2}, x_{3}\right)=c_{31} x_{1}+c_{32} x_{2}+c_{33} x_{3}, \\
\text { s.t } \quad A_{1} x_{1}+A_{2} x_{2}+A_{3} x_{3} \leq b, \\
\quad x_{1} \geq 0, x_{2} \geq 0, x_{3} \geq 0 .
\end{gathered}
$$

To begin with, the fuzzy goals and membership functions need to be specified by the decision- makers in order to apply interactive fuzzy programming. In this section, the membership functions and fuzzy goals are identified in accordance with relations (1), (2), and (3).

\subsection{An Interactive Fuzzy Programming Approach}

In this subsection, the interactive fuzzy programming approach introduced by Sakawa and Nishizaki (2009) is given to address Problem 7. In their interactive fuzzy approach, Problem 2 is formulated for Problem 7 to find an initial satisfactory solution. In addition, enough knowledge about the membership function values of the objectives are provided which help the decision-makers at levels 1 and 2 to identify minimal satisfaction degrees for selves. Additionally, suitable upper and lower bounds are specified for ratios $\Delta_{21}=\frac{\mu_{2}\left(z_{2}(x)\right)}{\mu_{1}\left(z_{1}(x)\right)}$ and $\Delta_{32}=\frac{\mu_{3}\left(z_{3}(x)\right)}{\mu_{2}\left(z_{2}(x)\right)}$ by decision-makers at levels 1 and 2 , respectively.

Let $\hat{\delta}_{1}$ and $\hat{\delta}_{2}$ be the minimal satisfaction degrees of the decision-makers at levels 1 and 2 , respectively. Moreover, assume that decision-maker at level 1 specifies $\Delta_{L 21}$ and $\Delta_{U 21}$ as the lower and upper bounds for $\Delta_{21}=\frac{\mu_{2}}{\mu_{1}}$, respectively, while decision-maker at the second level identifies $\Delta_{L 32}$ and $\Delta_{U 32}$ as the lower and upper bounds for $\Delta_{32}=\frac{\mu_{3}}{\mu_{2}}$, respectively.

With the assumption that the decision-makers at levels 1 and 2 are not satisfied with the solution obtained of Problem 2, the following problems need to be considered to achieve a satisfactory solution for which the decision-makers at the levels 1 and 2 reach their minimal satisfaction degrees $\hat{\delta}_{1}$ and $\hat{\delta}_{2}$ of selves. Moreover, the satisfaction degrees of the decision-makers at the levels are balanced. To do so, the following problem is solved first in which only levels 2 and 3 are considered. 
Problem 8

Maximize

$\lambda$

s.t

$$
\begin{gathered}
\mu_{2}\left(z_{2}(x)\right) \geq \hat{\delta}_{2}, \\
\mu_{32}\left(\Delta_{32}\right) \geq \hat{\delta}_{32}, \\
\mu_{3}\left(z_{3}(x)\right) \geq \lambda, \\
A_{1} x_{1}+A_{2} x_{2}+A_{3} x_{3} \leq b, \\
x_{1} \geq 0, x_{2} \geq 0, x_{3} \geq 0 .
\end{gathered}
$$

In the above problem, linear membership function $\mu_{32}$ is defined according to values $\Delta_{L 32}$ and $\Delta_{U 32}$ as follows:

$$
\mu_{32}\left(\Delta_{32}\right)=\left\{\begin{array}{cr}
0, & \Delta_{32} \leq \Delta_{L 32} \\
\frac{\Delta_{32}-\Delta_{L 32}}{\Delta_{U 32}-\Delta_{L 32}}, & \Delta_{L 32} \leq \Delta_{32} \leq \Delta_{U 32} \\
1 & \Delta_{32} \geq \Delta_{U 32}
\end{array} .\right.
$$

In addition, $\hat{\delta}_{32}$ is identified by decision-maker at level 2 as the minimal satisfaction degree of $\mu_{32}\left(\Delta_{32}\right)$.

If Problem 8 is not feasible, either values of $\hat{\delta}_{2}$ or $\hat{\delta}_{32}$ or values of $\hat{\delta}_{2}$ and $\hat{\delta}_{32}$ must be decreased by decision-maker at level 2 till Problem 8 attains feasibility.

Let $\tilde{x}$ be the solution obtained of Problem 8. Subsequently, the following problem is solved to achieve a satisfactory solution for Problem 7.

Problem 9

Maximize s.t

$$
\begin{gathered}
\mu_{1}\left(z_{1}(x)\right) \geq \hat{\delta}_{1}, \\
\mu_{21}\left(\Delta_{21}\right) \geq \hat{\delta}_{21}, \\
\mu_{2}\left(z_{2}(x)\right) \geq \lambda, \\
\mu_{3}\left(z_{3}(x)\right) \geq \lambda *\left(\frac{\mu_{3}\left(z_{3}(\tilde{x})\right)}{\mu_{2}\left(z_{2}(\tilde{x})\right)}\right) \\
A_{1} x_{1}+A_{2} x_{2}+A_{3} x_{3} \leq b, \\
x_{1} \geq 0, x_{2} \geq 0, x_{3} \geq 0 .
\end{gathered}
$$

In the above problem $\mu_{21}\left(\Delta_{21}\right)$ is defined as follows:

$$
\mu_{21}\left(\Delta_{21}\right)=\left\{\begin{array}{lr}
0, & \Delta_{21} \leq \Delta_{L 21} \\
\frac{\Delta_{21}-\Delta_{L 21}}{\Delta_{U 21}-\Delta_{L 21}}, & \Delta_{L 21} \leq \Delta_{21} \leq \Delta_{U 21} \\
1, & \Delta_{21} \geq \Delta_{U 32}
\end{array}\right.
$$

Additionally, $\hat{\delta}_{21}$ is a minimal satisfaction degree for $\mu_{21}\left(\Delta_{21}\right)$ specified by the decision-maker at the first level.

If Problem 9 loses feasibility, either values of $\hat{\delta}_{1}$ or $\hat{\delta}_{21}$ or values of $\hat{\delta}_{1}$ and $\hat{\delta}_{21}$ must be decreased by decision-maker at level 1 so as to Problem 9 becomes feasible.

The solution obtained of Problem 9 is a satisfactory solution for Problem 7 in the method introduced by Sakawa and Nishizaki (2009).

\subsection{Three-Level to Two-Level Program}

In this part, it is demonstrated that a three-level LPP with mutual cooperative relationship established between can be further DMs reduced into a two-level LPP with two DMs at the upper level and single DM at the lower level. The proposed method in section 3 can therefore be used to find a satisfactory solution instead of rather difficult proposed method in subsection 5.1 by Sakawa and Nishizaki (2009).

Similar to the previous subsection, Problem 2 is formulated for Problem 7 to find an initial satisfactory solution. This initial satisfactory solution provides a full knowledge of membership functions of the objectives for DMs at the levels 1 and 2 to specify minimal satisfaction degrees for selves in addition to identify lower and upper 
bounds for the ratios of satisfaction degrees.

Let $\hat{\delta}_{1}$ and $\hat{\delta}_{2}$ be the minimal satisfaction degrees of the DMs at levels 1 and 2, respectively. Moreover, assume that the DM at level 1 specifies $\Delta_{L 21}$ and $\Delta_{U 21}$ as the lower and upper bounds for $\Delta_{21}=\frac{\mu_{2}}{\mu_{1}}$, respectively, while DM at the second level respectively identifies $\Delta_{L 32}$ and $\Delta_{U 32}$ as the lower and upper bounds for $\Delta_{32}=\frac{\mu_{3}}{\mu_{2}}$.

According to the fact that DMs at levels 1 and 2 have minimal satisfaction degrees of selves and higher priority to the one at level 3, Problem 7 can therefore be transformed into two-level LPP. In the resulted two-level programming problem, decision-makers at the first and second levels of Problem 7 are considered as leaders and the one at the third level is considered as follower. As a consequence, Problem 7 is reduced into the following two-level linear programming problem in which there exists two DMs at the upper level and single DM at the lower level:

Problem 10

$$
\begin{aligned}
& \text { Minimize }_{\text {upper level(DM1) }} z_{1}\left(x_{1}, x_{2}, x_{3}\right)=c_{11} x_{1}+c_{12} x_{2}+c_{13} x_{3} \text {, } \\
& \underset{\text { upper level }(D M 2)}{\operatorname{Minimize}} z_{2}\left(x_{1}, x_{2}, x_{3}\right)=c_{21} x_{1}+c_{22} x_{2}+c_{23} x_{3} \text {, } \\
& \underset{\text { lower level(DM } 3)}{\operatorname{Minimize}} z_{3}\left(x_{1}, x_{2}, x_{3}\right)=c_{31} x_{1}+c_{32} x_{2}+c_{33} x_{3} \text {, } \\
& \text { s.t } \quad A_{1} x_{1}+A_{2} x_{2}+A_{3} x_{3} \leq b, \\
& x_{1} \geq 0, x_{2} \geq 0, x_{3} \geq 0 \text {. }
\end{aligned}
$$

Lower and upper bounds for the ratio of satisfaction degree of DM1 to satisfaction degree of DM3 must be identified in order to find a satisfactory solution to the above problem. To do this, lower bound $\Delta_{L 31}=\Delta_{L 32} \times \Delta_{L 21}$ and upper bound $\Delta_{U 31}=\Delta_{U 32} \times \Delta_{U 21}$ are accordingly obtained for $\Delta_{31}=\frac{\mu_{3}}{\mu_{1}}$ using relation $\frac{\mu_{3}}{\mu_{1}}=\frac{\mu_{3}}{\mu_{2}} \times \frac{\mu_{2}}{\mu_{1}}$.

In the above problem, DM1 and DM2 are decision-makers at the top level with minimal satisfaction degrees $\hat{\delta}_{1}$ and $\hat{\delta}_{2}$, respectively. Procedure 2 described in section 3 must accordingly be followed to update minimal satisfaction degrees $\hat{\delta}_{1}$ and $\hat{\delta}_{2}$ of decision-makers at the upper level in order to achieve a satisfactory solution. The termination conditions at iteration $l$ are therefore as following:

Condition 1: The minimal satisfaction levels of DM1 and DM2 must be greater than $\hat{\delta}_{1}$ and $\hat{\delta}_{2}$, respectively. (i.e., $\mu_{1}\left(z_{1}^{l}\right) \geq \hat{\delta}_{1}$ and $\left.\mu_{2}\left(z_{2}^{l}\right) \geq \hat{\delta}_{2}\right)$.

Condition 2: $\Delta_{\text {max }}^{l} \in\left[\Delta_{L}, \Delta_{U}\right]$ and $\Delta_{\min }^{l} \in\left[\Delta_{L}, \Delta_{U}\right]$, where $\left[\Delta_{L}, \Delta_{U}\right]=\left[\Delta_{L 31}, \Delta_{U 31}\right] \cap\left[\Delta_{L 32}, \Delta_{U 32}\right]$.

\section{Discussion}

The problem considered in this paper is the cooperative two-level LPP with two leaders and single follower. Two constraints related to the minimum satisfaction degrees of the leaders must be added to the feasible region of the problem in order to find the satisfactory solution in such a manner to be in favor of the leaders. While, only one constraint needs to be considered regarding the minimum satisfaction degree of the leader in the two-level LPP with single leader. Due to the fact that adding a more constraint to a feasible region make that feasible region smaller, therefore the feasible region of a two-level LPP with two leaders is smaller than the same problem with only one leader. Thus, the satisfactory solution obtained for two-level LPP with single leader and single follower is better than the same two-level LPP with two leaders. In other word, the average of the membership function's value of the objectives of the two-level LPP with two leaders is smaller than the average of the membership functions' values of the objectives of the same two-level LPP with only one leader.

\section{Conclusion}

In this paper, a method based on interactive fuzzy programming was introduced to obtain a satisfactory solution to the two-level linear programming problems with two decision-makers at the upper level and single-decision maker at the lower level when a mutually cooperative relationship exists between the decision-makers. For the obtained satisfactory solution, which is also a Pareto optimal solution, both leaders are satisfied, and an appropriate coordination exists between the satisfaction degree of the follower and the satisfaction degree of each leader. In addition, it was shown that a three-level program can be transformed into a two-level program with two decision-makers at the upper level. 


\section{Acknowledgments}

This work was supported by funding from FRGS/1/2013/SG04/UKM/02/2.

\section{References}

Abd El-Wahed, W. F., \& Lee, S. M. (2006). Interactive fuzzy goal programming for multi-objective transportation problems. Omega, 34, 158-166. http://dx.doi.org/10.1016/j.omega.2004.08.006

Anandalingam, G. (1988). A mathematical programming model of decentralized multi-level systems. Journal of the Operational Research Society, 39, 1021-1033. http://dx.doi.org/10.1057/jors.1988.172

Anandalingam, G., \& White, D. (1990). A solution method for the linear static Stackelbergproblem using penalty function. IEET Transactions on Autamatic Control, 35, 1170-1173.

Bard, J. F. (1984). An investigation of the linear three level programming problem. Systems, Man and Cybernetics, IEEE Transactions on, 5, 711-717.

Bard, J. F. (1998). Practical bilevel optimization: Algorithm and Applications. Kluwer Academic Publishers, Dordrecht.

Bard, J. F., \& Falk, J. E. (1982). An explicit solution to the multi-level programming problem. Comput.Oper. Res. 9, 77-100. http://dx.doi.org/10.1016/0305-0548(82)90007-7

Bard, J. F., \& Moore, J. T. (1990). A branch and bound algorithm for the bilevel programmingproblem. SIAM J.Sci. Stat. Comput, 11, 281-292. http://dx.doi.org/10.1137/0911017

Bellman, R. E., \& Zadeh, L. A. (1970). Decision making in a fuzzy environment. Manage. Sci, 17, 141-164.

Bialas, W. F., \& Karwan, M. H. (1984). Two-level linear programming. Manag. Sci., 30, 1004-1020.

Borza, M., Rambely, A. S., \& Saraj, M. (2012). A Stackelberg solution to a two-level linear fractional programming problem with interval coeeficients in the objective functions. Sains Malaysiana, 41, 1651-1656.

Hansen, P., Jaumard, B., \& Savard, G. (1992). New branch-and-bound rules for bilevelprogramming. SIAM J.Sci. Stat. Comput, 13, 1194-1217.

Lai, Y. J. (1996). Hierarchical optimization: a satisfactory solution. Fuzzy Sets and Systems, 77, 321-335. http://dx.doi.org/10.1016/0165-0114(95)00086-0

Migdalas, A., Pardalos, P. M., \& Varbrand, P. (1998). Multi-level Optimization: Algorithms and Applications. Kluwer Academic Publishers, Dordrecht.

Sakawa, M., \& Nishizaki, I. (2001). Interactive fuzzy programming for multi-level nonconvex nonlinear programming problems through genetic algorithms. Dynamical aspects in fuzzy decision making. In Y. Yoshida (Ed.), Phisical-Verlag, Heidelberg, Chapter, 5, 99-116.

Sakawa, M., \& Nishizaki, I. (2002). Interactive fuzzy programming for decentralized two-level linear programming problems. Fuzzy Sets and Systems, 125, 301-315. http://dx.doi.org/10.1016/S0165-0114(01)00042-2

Sakawa, M., \& Nishizaki, I. (2009). Cooperative and noncooperative multi-level programming. Speringer US. Pages 97-106.

Sakawa, M., Nishizaki, I., \& Uemura, Y. (1998). Interactive fuzzy programming for multi-level linear programming problems. Computer \& Mathematics with Applications, 36, 71-86. http://dx.doi.org/10.1016/s0898-1221(98)00118-7

Sakawa, M., Nishizaki, I., \& Uemura, Y. (2001). Interactive fuzzy programming for two-level linear and linear fractional production and assignment problems: a case study. European Journal of Operational Research, 135, 142-157. http://dx.doi.org/10.1016/S0377-2217(00)00309-X

Sakawa, M., Nishizaki, I., \& Uemura, Y. (2002). A decentralized two-level transportation problem in a housing material manufacturer: Interactive fuzzy programming approach. European Journal of Operational Research, 141, 167-185. http://dx.doi.org/10.1016/S0377-2217(01)00273-9

Sherali, H. (1984). A multiple leader Stackelberg model and analysis. Operations Research, 32, 390-404. Retrieved from http://www.jstor.org/stable/172107

Shih, H. S., Lai, Y. J., \& Lee, E. S. (1996). Fuzzy approach for multi-level programming problems. Computers and Operational Research, 23, 73-91. http://dx.doi.org/10.1016/0305-0548(95)00007-9 
Simaan, M., \& Cruz, J. B. (1973). A Stackelberg solution for games with many players. Automatic Control, IEEE Transactions on, 18, 322-324. http://dx.doi.org/10.1109/tac.1973.1100307

Stackelberg, H. V. (1934). Market form und Gleicgwicht. Springer-Verlag, Berlin.

Wen, U. P., \& Bialas, W. F. (1986). The hybrid algorithm for solving the three-level linear programming problem. Computers \& operations research, 13, 367-377. http://dx.doi.org/10.1016/0305-0548(86)90023-7

White, D., \& Anandalingam, G. (1993). A Penalty function approach for solving bi-level programs. J. Glob. Optim., 3, 397-419.

Zimmermann, H. J. (1978). Fuzzy programming and linear programming with several objective functions. Fuzzy Sets and Systems, 1, 45-55. http://dx.doi.org/10.1016/0165-0114(78)90031-3

\section{Copyrights}

Copyright for this article is retained by the author(s), with first publication rights granted to the journal.

This is an open-access article distributed under the terms and conditions of the Creative Commons Attribution license (http://creativecommons.org/licenses/by/3.0/). 